\title{
WRIDY: MULTISENSORY WRITING APPROACH IN MOBILE APPLICATION FOR KIDS WITH LEARNING DISABILITIES INCLUDING DYSLEXIA
}

\author{
T.N. Wee, R.U. Khan, Y.B. Oon, J.C. Lee \\ Universiti Malaysia Sarawak (UNIMAS) (MALAYSIA)
}

\begin{abstract}
Handwriting development begins as early as infancy when children are first able to grasp a writing object and leave a mark on the paper. Handwriting is linked with brain functioning; experts suggest that handwriting skill lightens a student's cognitive load. With consistent handwriting practice, it becomes less demanding and more automatic, enabling students critical thinking and thought organization. The lack of writing skill decreases kids' capacity to carry out higher-order skills. Most of the writing intervention approaches are not multisensory and some are using substances that may be dangerous for kids such as sand or shaving cream or pipe cleaners or play-doh etc. These issues become more challenging for kids with a learning disability such as dyslexia. This empirical gap in the multisensory writing system is the target of this research. A multisensory mobile application (Wridy) is designed and developed to support kids with learning disabilities. Wridy is an early-stage multisensory writing intervention tool. It uses a dyslexia-friendly user interface, fonts and colour. Wridy is demonstrated to the teachers of the Dyslexia Association Kuching, Malaysia. The results of the survey show that Wridy is helpful and useful in learning writing alphabets especially for kids with learning disabilities such as dyslexia.
\end{abstract}

Keywords: multisensory Intervention tools, Assistive technology, Multisensory, Learning disability, Dyslexia.

\section{INTRODUCTION}

The act of writing can be trace back thousands of years where our ancestors made markings on rocks, papyrus, papers and other various objects and surfaces. The aim of our ancestors was to record, communicate and preserve important information. Like our ancestors, writing is also an essential part of our daily lives. Out of school, writing is also an important part as writing will be a requirement in most of the jobs available. However, there are individuals that face difficulty writing on daily basis as more than 700 million children and adults worldwide are found to have dyslexia [1].

Dyslexia is a specific learning difficulty with neurodevelopmental disorder that affects literacy skills of an individual [1][2]. Individuals with dyslexia will experience problems in reading, spelling and writing. The problems stems from the difficulty in connecting and associating words with their corresponding sounds due to disruptions in brain regions responsible for language processing [3]. The uniqueness in the way their brain process language shows that literacy problems they faced were not caused by the lack in intellectual abilities and visual or audio impairment.

Among the difficulty that an individual with dyslexia faces, difficulty in writing is not as well-known as other literacy skill problems. Difficulty in reading and spelling often comes straight to mind when associating with dyslexia. Regardless, the literacy difficulties are interrelated as according to [4], difficulties in reading also contributes to difficulty in writing. Reading and writing undergo the same underlying process of decoding and encoding phonological information. Besides having the same underlying process, reading is heavily involved during the writing process as the ability to proofread one's writing is important to the learning process and quality of the writing.

To combat the difficulty, multisensory intervention approach is one of the common intervention methods used for individuals with dyslexia. The approach focuses on senses such as visual, auditory, tactile and kinaesthetic together with ways to incorporate and engage multiple senses appropriately during the learning process [5].

Some of the most common items used in multisensory intervention approaches are sandbox, sandpaper, whipping or shaving cream, and playdoh. Even though those items provide multisensory experience, the child need to be monitored as the items can be messy and even hazardous when ingested. Learning Without Tears developed Handwriting Without Tears a curriculum that incorporate 